\title{
Consultas ambulatorias pediátricas atendidas en el Servicio de Urgencia de un hospital universitario
}

\author{
BÁRBARA LARA H. ${ }^{1}$, PABLO AGUILERA F. ${ }^{1}$, MARCELA GARRIDO V. ${ }^{1}$, \\ TAMARA HIRSCH B. ${ }^{2}$, STUART SWADRON ${ }^{3}$, FERNANDO SALDÍAS P. ${ }^{1}$ \\ 1. Programa de Medicina de Urgencia. Departamento de Medicina, Pontificia Universidad Católica de Chile. \\ 2. Programa de Medicina de Urgencia. Departamento de Pediatría, Pontificia Universidad Católica de Chile. \\ 3. Department of Emergency Medicine, University of Southern California, Los Angeles, USA.
}

\begin{abstract}
Pediatric outpatient consultation at the Emergency Department of a University Hospital
\end{abstract}

Introduction: To determine the epidemiological profile of pediatric consultations treated at the emergency department (ED) is essential for planning processes of medical care and to guide education programs and research. Objectives: To describe the characteristics of the child population and the main reasons for consultation (RFC) seen in a pediatric emergency service. Patients and Method: A retrospective, descriptive clinical study was conducted regarding the visits to the Children's Emergency Service of an academic hospital in Santiago, for a period of twelve months. RFC were analyzed by age group, severity, seasonality, disposition and frequency of recurrent visits. Results: 24,531 pediatric consultations were evaluated, $51.9 \%$ were male $(\mathrm{n}=12,720)$. The age of the patients ranged between one day old and 15 years, with a median age of 36.5 months. $1.5 \%$ of patients were newborns $(\mathrm{NB}), 17.6 \%$ were infants $(\mathrm{n}=4,326), 51.9 \%$ were preschoolers $(\mathrm{n}=12,725)$ and $29 \%$ were school children $(\mathrm{n}=7,118)$. Major RFC were fever $(\mathrm{n}=6,643,28.2 \%)$, gastrointestinal symptoms $(n=5,606,23.8 \%)$ and respiratory symptoms $(n=5,018,21.3 \%)$, which did not differ significantly according to gender. Most patients $(95.5 \%)$ were sent to their homes. The risk of hospitalization was more elevated in NB and in those with jaundice $(\mathrm{OR}=7.20,95 \%$ CI 3.12 to 16.6$)$, neurological symptoms $(\mathrm{OR}=6.90,95 \% \mathrm{CI} 4.60-10.4)$ and poisoning $(\mathrm{OR}=6.45,95 \% \mathrm{CI} 2.82$ to 14.7$)$. About $4 \%$ were repeat visits, especially in the NB group. Conclusions: The epidemiological profile of pediatric consultations seen at the ED was similar to that described in previous studies. However, a lower rate of hospitalization was found even though the patients had similar risk profile.

(Key words: Emergency department, triage, hospitalization, pediatrics, epidemiology).

Rev Chil Pediatr 2014; 85 (2): 174-182

Recibido el 3 de abril de 2013, devuelto para corregir el 10 de julio de 2013, segunda versión 9 de septiembre de 2013, aceptado para publicación el 28 de noviembre de 2013.

Este trabajo cumple con los requisitos sobre consentimiento /asentimiento informado, comité de ética, financiamiento, estudios animales y sobre la ausencia de conflictos de intereses según corresponda.

Correspondencia a:

Dr. Fernando Saldías Peñafiel

E-mail: fsaldias@med.puc.cl 


\section{RESUMEN}

Introducción: Conocer el perfil epidemiológico de las consultas pediátricas atendidas en el servicio de urgencia (SU) es esencial para planificar los procesos de atención médica y orientar los programas de educación e investigación. Objetivos: Describir las características de la población infantil y los principales motivos de consulta (MC) atendidos en un SU pediátrico. Pacientes y Método: Estudio clínico descriptivo retrospectivo de las visitas realizadas a un SU infantil en un hospital académico de Santiago durante un período de doce meses. Se analizaron los MC por grupo etario, gravedad, estacionalidad, forma de egreso y frecuencia de visitas recurrentes. Resultados: Se evaluaron 24.531 consultas pediátricas, 51,9\% $(\mathrm{n}=12.720)$ eran varones. La edad de los pacientes osciló entre un día y 15 años, con una mediana de 36,5 meses. El 1,5\% de los pacientes ( $\mathrm{n}=$ $362)$ eran recién nacidos $(\mathrm{RN}), 17,6 \%(\mathrm{n}=4.326)$ lactantes, $51,9 \%(\mathrm{n}=12.725)$ preescolares y $29 \%(\mathrm{n}=7.118)$ escolares. Los principales MC fueron fiebre $(n=6.643,28,2 \%)$, síntomas gastrointestinales $(n=5.606,23,8 \%)$ y síntomas respiratorios $(\mathrm{n}=5.018,21,3 \%)$, los cuales no difirieron significativamente según género. La mayoría de los pacientes $(95,5 \%)$ fueron enviados a su domicilio. El riesgo de hospitalización fue más elevado en los RN y en aquellos que consultaron por ictericia ( $\mathrm{OR}=7,20$; IC 95\% 3,12-16,6), síntomas neurológicos (OR $=6,90$; IC 95\% 4,60-10,4) e intoxicaciones $(\mathrm{OR}=6,45$; IC 95\% 2,82-14,7). Alrededor del 4\% fueron consultas repetidas, especialmente en los RN. Conclusiones: El perfil epidemiológico de las consultas pediátricas atendidas en el SU fue similar al descrito en estudios internacionales. Sin embargo, encontramos una menor tasa de hospitalización a pesar que los pacientes presentaban un perfil de riesgo similar en la admisión al SU. (Palabras clave: Servicio de urgencia, triage, hospitalización, pediatría, epidemiología).

Rev Chil Pediatr 2014; 85 (2): 174-182

\section{Introducción}

El Programa de formación en Medicina de Urgencia, incluyendo su rama pediátrica, es una especialidad médica que se ha desarrollado en países del hemisferio norte desde hace cuatro décadas $^{1-3}$; en nuestro país se encuentra en las etapas iniciales de desarrollo, existiendo al menos cinco programas de formación universitaria con énfasis en la atención de población adulta. Conocer las características de los pacientes que asisten a los servicios de urgencia (SU), sus principales motivos de consulta y el nivel de gravedad o complejidad de los mismos, es esencial para administrar estos servicios y los hospitales de los que forman parte, para diseñar e implementar los programas de formación del equipo de salud y, además, constituiría el sustrato para la planificación de estrategias en salud pública que faciliten el acceso, manejo y prevención de los problemas de salud que afligen a la población.

El análisis de la información epidemiológica ha permitido en otros países diseñar e implementar sistemas de estratificación de riesgo (triage), algoritmos de estudio y protocolos de manejo en base a los motivos de consulta más comunes atendidos en los servicios de urgencia $^{4}$. Como parte de un programa académico de medicina de urgencia, el conocimiento de la epidemiología y principales motivos de consulta es esencial para enfocar el currículo académico $^{2}$. Cada uno de estos escenarios lleva a un potencial diagnóstico operativo y valoración de severidad. Los residentes y alumnos de pregrado de medicina y enfermería debiesen dominar el enfrentamiento y manejo riguroso de estos temas.

Por otro lado, esta información puede ser de utilidad en el momento de evaluar los requerimientos y la asignación de los recursos humanos y materiales basados en la realidad local. Sin embargo, la mayoría de los datos existentes de SU pediátricos provienen de poblaciones extranjeras, principalmente norteamericana y europea ${ }^{5-7}$. Además, la gran mayoría de los estudios internacionales se basan en la descripción de SU generales, describiendo sólo tangencialmente a la subpoblación pediátrica, mientras que otros enfocan el análisis en categorías de riesgo específicas, principalmente recién nacidos $y$ adolescentes ${ }^{8-12}$. La litera- 
tura nacional es escasa ${ }^{13-15}$, lo que nos ha motivado a colectar y analizar los datos disponibles de nuestro SU, con el fin de aportar con datos más acotados a nuestra realidad.

El objetivo de este estudio es analizar los principales motivos de consulta en el SU pediátrico de un hospital académico, urbano y de alta complejidad, de la Región Metropolitana. Con este estudio esperamos facilitar y motivar el diseño y la planificación de nuevos proyectos de investigación tanto en éste como en otros establecimientos nacionales.

\section{Pacientes y Método}

\section{Diseño}

Análisis retrospectivo de todas las visitas al SU pediátrico del Hospital Clínico de la Pontificia Universidad Católica de Chile (HCPUC) durante doce meses consecutivos, desde el 1 de noviembre de 2009 hasta el 31 de octubre de 2010. El inicio del estudio fue elegido deliberadamente al término de la pandemia de influenza H1N1 que afectó a nuestro país. El HCPUC es un hospital académico de 520 camas, 12\% pediátricas, ubicado en el centro de Santiago, de alta complejidad y centro de referencia nacional para pacientes con malformaciones cardíacas congénitas. El hospital tiene 59 camas pediátricas, con 19 camas básicas, 4 de mediana complejidad, 12 de alta complejidad y 24 de cuidados críticos neonatológicos. El SU pediátrico consta de diez boxes de atención de urgencia indiferenciada. Todos los pacientes de edad igual o menor a 15 años son atendidos en la sección pediátrica del SU y fueron incluidos en este estudio. Los pacientes pediátricos que consultaron directamente al SU de traumatología y ginecología no fueron incluidos. Este protocolo fue aprobado por el Comité de Ética de la Institución y se solicitó la dispensa de consentimiento informado por tratarse de un estudio clínico descriptivo no intervencional donde se protegió la confidencialidad de la información clínica obtenida de los pacientes.

\section{Recolección de datos}

Los datos epidemiológicos y clínicos fueron extraídos de los registros clínicos consignados por el equipo de salud y el personal administrativo del servicio en el momento de la consulta. Con un objetivo operacional, definimos como primer y segundo motivo de consulta a los dos primeros síntomas o signos que determinaron la visita al SU declarados por el cuidador y/o el paciente en el momento del triage. De cada consulta se extrajeron los siguientes datos: fecha, edad, género, modalidad de ingreso, principales motivos de consulta, destino y severidad. La gravedad del paciente en la admisión fue definida mediante el Índice de Gravedad de Emergencia (Emergency Severity Index, ESI), registrado en el momento del triage por una enfermera especializada ${ }^{16}$. En el análisis de la información, los pacientes fueron agrupados en los siguientes grupos etarios: Recién nacidos (RN) (1-28 días), lactantes (29 días1 año), lactante mayores y preescolares (2-5 años), escolares y adolescentes (6 años y más). Los motivos de consulta se categorizaron por sistemas. Las consultas repetidas fueron definidas como aquellas visitas realizadas dentro de las primeras $72 \mathrm{~h}$ posteriores a la consulta original. El primer motivo de consulta de cada visita fue analizado según edad, género, gravedad (ESI), destino, consulta repetida y patrón estacional. El segundo motivo de consulta fue analizado sólo cuando el primero era fiebre.

\section{Análisis estadístico}

Los resultados fueron expresados como valores numéricos y porcentajes para las medidas en escala nominal. Las variables cualitativas fueron comparadas mediante la prueba de $\chi^{2}$. Como estimador de gravedad se calcularon las razones o cocientes de probabilidad (odds ratio e intervalos de confianza de 95\%) de hospitalización de los diferentes motivos de consulta. Para ello se utilizaron los programas Epi-Info 6.0 (CDC, Atlanta) e IBM SPSS Statistics 19.0 (SPSS Inc., Chicago).

\section{Resultados}

Durante el período del estudio se evaluaron 24.531 consultas pediátricas en el SU del HCPUC, correspondiendo al 31,3\% de todas las visitas al SU general. Los motivos de con- 
sulta se categorizaron por sistemas (tabla 1). El perfil demográfico de los pacientes se describe en la tabla 2. La edad de los pacientes fluctuó entre 1 día y 15 años, con una mediana de 36,5 meses y una media de 51 meses ( $D E=45,6$ meses $)$. El 1,5\% $(n=362)$ de las visitas fueron hechas por $\mathrm{RN}$, el $17,6 \%(\mathrm{n}=4.326)$ por lactantes, el $51,9 \%(n=12.725)$ por lactantes mayores y preescolares y el $29 \%(n=7.118)$ por escolares y adolescentes. Cerca de la mitad de las visitas $(n=12.720 ; 51,9 \%)$ fueron realizadas por varones. La mayoría de los pacientes correspondieron a las categorías de riesgo 3 y 4 $(92,7 \%, \mathrm{n}=22.754)$, y sólo algunos pacientes requirieron atención inmediata (ESI 2: 2,1\%) o medidas de reanimación (ESI 1: 0,04\%). Los principales motivos de consulta en los pacientes graves (ESI 1 y 2) fueron síntomas respiratorios y neurológicos.

Los principales motivos de consulta en el SU fueron fiebre $(n=6.643 ; 28,2 \%)$, síntomas gastrointestinales $(n=5.606 ; 23,8 \%)$, síntomas respiratorios altos y bajos $(\mathrm{n}=5.018 ; 21,3 \%)$, cefalea $(\mathrm{n}=1.235 ; 5,2 \%)$ y síntomas relacionados a afecciones de la piel $(\mathrm{n}=1.119 ; 4,8 \%)$ (tabla 3). En el período neonatal, la ictericia $(8,3 \%)$ y síntomas generales $(22,2 \%)$ fueron más frecuentes que en cualquier otra categoría de edad. La cefalea (13\%) y dolor abdominal $(17,4 \%)$ fueron especialmente importantes entre escolares y adolescentes.

La mayoría de los pacientes $(95,5 \%)$ fueron dados de alta del SU, el $4 \%$ fue hospitalizado y $0,5 \%$ derivado a otra institución debido a la falta de disponibilidad de camas en el HCPUC, por motivos administrativos $\mathrm{u}$ otras razones no especificadas. No hubo fallecidos en el SU pediátrico durante el período estudiado. Entre los pacientes hospitalizados $(\mathrm{n}=932)$, los principales motivos de consulta fueron los síntomas digestivos $(\mathrm{n}=268,31,3 \%)$, fiebre $(\mathrm{n}=$ $198,23,2 \%)$ y síntomas respiratorios $(\mathrm{n}=195$,

\section{Tabla 1. Clasificación de los motivos de consulta* atendidos en el servicio de urgencia pediátrico}

\begin{tabular}{|c|c|}
\hline Categorías & Síntomas incluidos en cada categoría \\
\hline Gastrointestinales & $\begin{array}{l}\text { Dolor abdominal, náuseas, vómitos, diarrea, constipación, distensión abdominal, flatulencias, hemate- } \\
\text { mesis, melena, hematoquezia, otras alteraciones de las deposiciones }\end{array}$ \\
\hline $\begin{array}{l}\text { Síntomas respiratorios } \\
\text { altos y bajos }\end{array}$ & $\begin{array}{l}\text { Congestión nasal, rinorrea, odinofagia, descarga nasal posterior, disfonía, afonía, otalgia, otorrea, } \\
\text { otros síntomas y signos del sistema auditivo. Disnea, dolor torácico, falta de aire, tos, expectoración, } \\
\text { hemoptisis }\end{array}$ \\
\hline $\begin{array}{l}\text { Síntomas relacionados } \\
\text { a afecciones de la piel }\end{array}$ & Rash, alteraciones de la piel, dermatitis del pañal, dermatitis de contacto \\
\hline Síntomas generales & Fatiga, irritabilidad, mialgias, Ilanto excesivo o inconsolable \\
\hline Genitourinarios & $\begin{array}{l}\text { Disuria, urgencia urinaria, incontinencia urinaria, retención urinaria, hematuria, dolor genital, secreción } \\
\text { uretral, flujo vaginal, dolor lumbar, otros síntomas genitourinarios no especificados }\end{array}$ \\
\hline Lesiones y trauma & Heridas, contusiones, fracturas, esguinces, luxaciones, quemaduras, cuerpos extraños \\
\hline Intoxicaciones & $\begin{array}{l}\text { Intoxicaciones voluntarias e involuntarias; ingesta de medicamentos, alcohol, drogas ilícitas, productos } \\
\text { del hogar; intoxicación por monóxido de carbono }\end{array}$ \\
\hline Síntomas neurológicos & $\begin{array}{l}\text { Convulsiones, movimientos involuntarios, alteraciones motoras, compromiso de conciencia, coma, otros } \\
\text { síntomas y signos neurológicos no clasificados }\end{array}$ \\
\hline Oftalmológicos & $\begin{array}{l}\text { Secreción ocular, ojo rojo, dolor ocular, disminución de la agudeza visual, signos y síntomas de las } \\
\text { pestañas, otros síntomas y signos no especificados relacionados a la visión }\end{array}$ \\
\hline Cardiovasculares & Disnea, ortopnea, DPN, dolor torácico, síncope, palpitaciones, edema \\
\hline Ictericia & Ictericia \\
\hline Cefalea & Dolor de cabeza, cefalea \\
\hline Fiebre & Fiebre, calofríos \\
\hline
\end{tabular}

* Los motivos de consulta fueron clasificados en base a la experiencia clínica de los autores y las categorías más frecuentemente encontradas en la literatura. La revisión de la literatura se realizó en pubmed con las siguientes palabras claves: chief complaints, reason for visits, emergency department, pediatrics, children. 
Tabla 2. Características clínicas de las 24.531 consultas pediátricas atendidas en el SU del Hospital Clínico de la Pontificia Universidad Católica de Chile

\begin{tabular}{|c|c|c|c|}
\hline Características & Categorías & $\mathbf{n}$ & $\%$ \\
\hline Grupos etarios & $\begin{array}{l}1 \text { a } 28 \text { días (RN) } \\
29 \text { días a } 1 \text { año (Lactantes) } \\
1 \text { a } 5 \text { años (Preescolares) } \\
6 \text { años y más (Escolares) }\end{array}$ & $\begin{array}{r}362 \\
4.326 \\
12.725 \\
7.118\end{array}$ & $\begin{array}{r}1,5 \\
17,6 \\
51,9 \\
29,0\end{array}$ \\
\hline Género & $\begin{array}{l}\text { Femenino } \\
\text { Masculino }\end{array}$ & $\begin{array}{l}11.811 \\
12.720\end{array}$ & $\begin{array}{l}48,1 \\
51,9\end{array}$ \\
\hline Categorías de riesgo en el triage & $\begin{array}{l}\mathrm{T} 1 \\
\mathrm{~T} 2\end{array}$ & $\begin{array}{r}11 \\
520\end{array}$ & $\begin{array}{l}0 \\
2,1\end{array}$ \\
\hline (Emergency Severity Index) & $\begin{array}{l}\text { T3 } \\
\text { T4 } \\
\text { T5 }\end{array}$ & $\begin{array}{r}6.436 \\
16.318 \\
1.246\end{array}$ & $\begin{array}{r}26,2 \\
66,5 \\
5,1\end{array}$ \\
\hline Modalidad de ingreso al servicio de urgencia ${ }^{1}$ & $\begin{array}{l}\text { Acompañado por cuidador } \\
\text { Solo } \\
\text { Ambulancia }\end{array}$ & $\begin{array}{r}22.322 \\
12 \\
76\end{array}$ & $\begin{array}{r}99,61 \\
0,05 \\
0,34\end{array}$ \\
\hline Destino $^{2}$ & $\begin{array}{l}\text { Enviado a su domicilio } \\
\text { Fallecidos } \\
\text { Hospitalización } \\
\text { Traslado a otro centro }\end{array}$ & $\begin{array}{r}22.451 \\
0 \\
932 \\
134\end{array}$ & $\begin{array}{l}95,5 \\
0 \\
4,0 \\
0,5\end{array}$ \\
\hline Total & & 24.531 & 100 \\
\hline
\end{tabular}

${ }^{1}$ En $8,6 \%$ de las visitas no se consignó la modalidad de ingreso al servicio de urgencia, por lo que no fueron incluidos en este análisis. ${ }^{2}$ En $4,1 \%$ de las visitas no estaba consignado el destino por lo que no fueron incluidos en este análisis.

Tabla 3. Motivos de consulta atendidos en el SU pediátrico por género y categorías de edad

\begin{tabular}{|c|c|c|c|c|c|c|c|c|c|c|c|c|c|c|}
\hline \multirow{3}{*}{$\begin{array}{l}\text { Motivo de } \\
\text { consulta }\end{array}$} & \multirow{2}{*}{\multicolumn{2}{|c|}{$\begin{array}{c}\text { Consultas } \\
\text { en servicio } \\
\text { urgencia }\end{array}$}} & \multicolumn{4}{|c|}{ Género } & \multicolumn{8}{|c|}{ Grupos etarios } \\
\hline & & & \multicolumn{2}{|c|}{ Masculino } & \multicolumn{2}{|c|}{ Femenino } & \multicolumn{2}{|c|}{$\mathbf{R N}$} & \multicolumn{2}{|c|}{ Lactantes } & \multicolumn{2}{|c|}{ Preescolares } & \multicolumn{2}{|c|}{ Escolares } \\
\hline & $\mathbf{n}$ & $\%$ & $\mathbf{n}$ & $\%$ & $\mathbf{n}$ & $\%$ & $\mathbf{n}$ & $\%$ & $\mathbf{n}$ & $\%$ & $\mathbf{n}$ & $\%$ & $\mathbf{n}$ & $\%$ \\
\hline Fiebre & 6.643 & 28,2 & 3.402 & 27,8 & 3.241 & 28,6 & 16 & 5,3 & 1.298 & 31,7 & 4.057 & 33,0 & 1.272 & 18,5 \\
\hline $\mathrm{GI}^{*}$ & 5.606 & 23,8 & 2.886 & 23,6 & 2.720 & 24,0 & 56 & 18,5 & 789 & 19,3 & 2.786 & 22,7 & 1.975 & 28,7 \\
\hline SRAB** & 5.018 & 21,3 & 2.735 & 22,3 & 2.283 & 20,2 & 93 & 30,8 & 1.326 & 32,4 & 2.580 & 21,0 & 1.019 & 14,8 \\
\hline Cefalea & 1.235 & 5,2 & 619 & 5,1 & 616 & 5,4 & 0 & 0 & 19 & 0,5 & 319 & 2,6 & 897 & 13,0 \\
\hline Piel y mucosas & 1.119 & 4,8 & 574 & 4,7 & 545 & 4,8 & 17 & 5,6 & 148 & 3,6 & 617 & 5,0 & 337 & 4,9 \\
\hline Lesión/trauma & 1.024 & 4,4 & 568 & 4,6 & 456 & 4,0 & 1 & 0,3 & 106 & 2,6 & 600 & 4,9 & 317 & 4,6 \\
\hline No clasificables & 892 & 3,8 & 492 & 4,0 & 400 & 3,5 & 18 & 6,0 & 85 & 2,1 & 447 & 3,6 & 342 & 5,0 \\
\hline S. generales & 920 & 3,9 & 471 & 3,8 & 449 & 4,0 & 67 & 22,2 & 247 & 6,0 & 315 & 2,6 & 291 & 4,2 \\
\hline Genitourinario & 577 & 2,4 & 216 & 1,8 & 361 & 3,2 & 5 & 1,7 & 15 & 0,4 & 318 & 2,6 & 239 & 3,5 \\
\hline Oftalmológicos & 200 & 0,8 & 111 & 0,9 & 89 & 0,8 & 2 & 0,7 & 30 & 0,7 & 109 & 0,9 & 59 & 0,9 \\
\hline Neurológicos & 149 & 0,6 & 77 & 0,6 & 72 & 0,6 & 1 & 0,3 & 18 & 0,4 & 85 & 0,7 & 45 & 0,6 \\
\hline Cardiovascular & 108 & 0,5 & 52 & 0,4 & 56 & 0,5 & 0 & 0 & 2 & 0 & 18 & 0,1 & 88 & 1,2 \\
\hline Intoxicaciones & 36 & 0,2 & 12 & 0,1 & 24 & 0,2 & 1 & 0,3 & 1 & 0 & 28 & 0,2 & 6 & 0,1 \\
\hline Ictericia & 33 & 0,1 & 24 & 0,2 & 9 & 0,1 & 25 & 8,3 & 5 & 0,1 & 2 & 0 & 1 & 0 \\
\hline Total*** & 23.560 & 100 & 12.239 & 100 & 11.321 & 100 & 302 & 100 & 4.089 & 100 & 12.281 & 100 & 6.888 & 100 \\
\hline Datos perdidos & 971 & 4,0 & 481 & 3,8 & 490 & 4,1 & 60 & 16,6 & 237 & 5,5 & 443 & 3,5 & 231 & 3,2 \\
\hline
\end{tabular}

*Gastrointestinales; **Síntomas respiratorios altos y bajos; ***el $4 \%$ de los motivos de consulta no estaban consignados en la base de datos y no fueron incluidos en el cálculo de los porcentajes. 
$22,8 \%$ ). El grupo con la mayor tasa de hospitalización fueron los RN ( $\mathrm{n}=49,14,4 \%$ de las visitas), especialmente con síntomas respiratorios $(n=12,30 \%)$ e ictericia $(n=6,15 \%)$. Entre los lactantes hospitalizados predominaron la fiebre y síntomas respiratorios $(\mathrm{n}=72$, $39,6 \%$ y $n=55,30,2 \%$, respectivamente) y en la población de lactantes mayores y preescolares fue la fiebre $(\mathrm{n}=106,28,8 \%)$ y los síntomas digestivos $(n=97,26,4 \%)$. La mayoría de los escolares hospitalizados fue debido a síntomas digestivos $(n=135,50,9 \%)$.

De acuerdo a las categorías de riesgo, la mayoría de los pacientes con ESI 1 fueron hospitalizados o referidos a otro centro; mientras que los pacientes en las categorías ESI 2 y 3 fueron admitidos o trasladados a otro hospital en el $39,2 \%$ y $10,1 \%$ de los casos, respectivamente. Los pacientes en categorías de riesgo ESI 4 y 5 fueron enviados a su hogar en el 98,5\% y el $99,5 \%$ de los casos, siendo ingresados el 1,5 y $0,5 \%$ de los casos, respectivamente.

Los motivos de consulta de los pacientes asociados a mayor riesgo de hospitalización fueron: Ictericia (OR = 7,20; IC 95\% 3,12$16,6)$, síntomas neurológicos (OR $=6,90$; IC $95 \% 4,60-10,4)$ e intoxicaciones $(\mathrm{OR}=6,45$; IC 95\% 2,82-14,7) (tabla 4).

El mayor número de visitas se concentró en los meses de invierno, especialmente debido a fiebre o síntomas respiratorios (tabla 5). En esa época del año, las afecciones respiratorias fueron el principal motivo de hospitalización $(34,2 \%$ de los ingresos en invierno) y su frecuencia aumentó en comparación con otras épocas del año. Los pacientes atendidos por síntomas respiratorios durante el invierno fueron hospitalizados más frecuentemente que aquellos atendidos durante el verano $(5,2 \% \mathrm{vs}$ 2,9\%). Casi la mitad de los pacientes atendidos por fiebre durante el invierno $(51,8 \%)$ tenían como segundo motivo de consulta síntomas respiratorios; lo cual aconteció sólo en el 19,3\% de las visitas durante el verano. Las lesiones traumáticas y los accidentes fueron más prevalentes durante las estaciones de verano y otoño $(\sim 5-6 \%)$.

De todas las visitas analizadas, un $3,9 \%$

Tabla 4. Motivos de consulta atendidos en el SU pediátrico y sus tasas de hospitalización

\begin{tabular}{|c|c|c|c|c|c|}
\hline $\begin{array}{l}\text { Motivos de consulta } \\
\text { en servicio urgencia }\end{array}$ & $\begin{array}{l}\text { n de } \\
\text { consultas }\end{array}$ & $\begin{array}{c}n \text { de } \\
\text { hospitalizaciones }\end{array}$ & $\%$ & $\begin{array}{c}\text { Riesgo de hospitalización } \\
\text { OR (IC95\%) }\end{array}$ & $\mathbf{p}$ \\
\hline Fiebre & 6.643 & 198 & 23,2 & $0,76 \quad(0,65-0,89)$ & $<0,001$ \\
\hline Gastrointestinales & 5.606 & 268 & 31,3 & $1,49 \quad(1,28-1,72)$ & $<0,001$ \\
\hline Síntomas respiratorios & 5.018 & 195 & 22,8 & $1,10 \quad(0,93-1,28)$ & 0,273 \\
\hline Cefalea & 1.235 & 21 & 2,5 & $0,45 \quad(0,29-0,69)$ & $<0,001$ \\
\hline Piel y mucosas & 1.119 & 20 & 2,3 & $0,47 \quad(0,30-0,74)$ & $<0,001$ \\
\hline Traumatismos & 1.024 & 9 & 1,1 & $0,23 \quad(0,12-0,44)$ & $<0,001$ \\
\hline Generales & 920 & 29 & 3,4 & $0,86 \quad(0,59-1,25)$ & 0,431 \\
\hline Genitourinarios & 577 & 14 & 1,6 & $0,66 \quad(0,39-1,12)$ & 0,121 \\
\hline Oftalmológicos & 200 & 4 & 0,5 & $0,54 \quad(0,20-1,45)$ & 0,223 \\
\hline Neurológicos & 149 & 30 & 3,5 & $6,90 \quad(4,60-10,4)$ & $<0,001$ \\
\hline Cardiovasculares & 108 & 5 & 0,6 & $1,29 \quad(0,53-3,17)$ & 0,578 \\
\hline Intoxicaciones & 36 & 7 & 0,8 & $6,45 \quad(2,82-14,7)$ & $<0,001$ \\
\hline Ictericia & 33 & 7 & 0,8 & $7,20 \quad(3,12-16,6)$ & $<0,001$ \\
\hline No clasificables & 892 & 48 & 5,6 & $1,54 \quad(1,14-2,08)$ & 0,0046 \\
\hline Total* & 23.560 & 855 & 100 & & \\
\hline Datos perdidos & 971 & 77 & 8,2 & & \\
\hline
\end{tabular}

*El 8,2\% de los motivos de consulta no estaban disponibles en los pacientes hospitalizados y no fueron incluidos en el cálculo de los porcentajes y odds ratios (OR). 
Tabla 5. Principales motivos de consulta atendidos en el SU pediátrico y su tasa de hospitalización según estaciones del año

\begin{tabular}{|c|c|c|c|c|c|c|}
\hline \multirow[b]{2}{*}{$\begin{array}{l}\text { Estación del } \\
\text { año }\end{array}$} & \multirow[b]{2}{*}{$\begin{array}{l}\text { Motivo de } \\
\text { consulta }\end{array}$} & \multicolumn{2}{|c|}{ Consultas } & \multicolumn{3}{|c|}{ Hospitalizaciones } \\
\hline & & $\mathbf{n}$ & $\%$ & n & $\%$ & $\begin{array}{c}\text { Tasa de } \\
\text { hospitalización }\end{array}$ \\
\hline \multirow[t]{6}{*}{ Invierno } & Fiebre & 2.535 & 34,5 & 79 & 28,4 & 3,12 \\
\hline & SRAB & 1.834 & 24,9 & 95 & 34,2 & 5,18 \\
\hline & $\mathrm{Gl}$ & 1.127 & 15,3 & 57 & 20,5 & 5,06 \\
\hline & Cefalea & 481 & 6,5 & 4 & 1,4 & 0,83 \\
\hline & Generales & 370 & 5,0 & 12 & 4,3 & 3,24 \\
\hline & Total* & 7.354 & & 278 & & \\
\hline \multirow[t]{6}{*}{ Primavera } & Gl & 1.744 & 28,0 & 85 & 36,3 & 4,87 \\
\hline & Fiebre & 1.742 & 27,9 & 54 & 23,1 & 3,10 \\
\hline & SRAB & 1.159 & 18,6 & 39 & 16,7 & 3,36 \\
\hline & Cefalea & 333 & 5,3 & 11 & 4,7 & 3,30 \\
\hline & Piel & 304 & 4,9 & 5 & 2,1 & 1,64 \\
\hline & Total* & 6.234 & & 234 & & \\
\hline \multirow[t]{6}{*}{ Verano } & Gl & 1.279 & 31,5 & 59 & 40,7 & 4,61 \\
\hline & Fiebre & 948 & 23,4 & 28 & 19,3 & 2,95 \\
\hline & SRAB & 592 & 14,6 & 17 & 11,7 & 2,87 \\
\hline & Piel & 279 & 6,9 & 4 & 2,8 & 1,43 \\
\hline & Traumatismos & 264 & 6,5 & 2 & 1,4 & 0,75 \\
\hline & Total* & 4.057 & & 145 & & \\
\hline \multirow[t]{6}{*}{ Otoño } & GI & 1.456 & 24,6 & 67 & 33,8 & 4,60 \\
\hline & SRAB & 1.433 & 24,2 & 44 & 22,2 & 3,07 \\
\hline & Fiebre & 1.418 & 24,0 & 37 & 18,7 & 2,61 \\
\hline & Piel & 297 & 5,0 & 7 & 3,5 & 2,36 \\
\hline & Traumatismos & 283 & 4,8 & 3 & 1,5 & 1,06 \\
\hline & Total* & 5.915 & & 198 & & \\
\hline
\end{tabular}

*En los valores totales se excluyen los motivos de consulta no consignados. GI: Síntomas gastrointestinales; SRAB: Síntomas respiratorios altos y bajos.

$(\mathrm{n}=938)$ fueron consultas repetidas, especialmente en niñas $(54,6 \%)$ en la etapa preescolar $(24,7 \%)$ y escolar $(26,8 \%)$. Sin embargo, la mayor tasa de consultas repetidas se encontró en el grupo de $\mathrm{RN}(8 \%$ de las visitas de $\mathrm{RN})$, seguido por lactantes $(5,3 \%)$, escolares $\mathrm{y}$ adolescentes $(3,5 \%)$ y lactantes mayores y preescolares $(3,3 \%)$. El patrón de distribución de los motivos de consulta fue muy similar al descrito en las consultas generales: Fiebre (35\%), síntomas digestivos $(31,3 \%)$, síntomas respiratorios $(17,5 \%)$ y afecciones de la piel $(6,2 \%)$. El promedio de consultas repetidas fue de 2,16 visitas por paciente.

\section{Discusión}

Los principales hallazgos de este estudio fueron: a) un tercio de las consultas atendidas en el SU fueron otorgadas a pacientes pediá- tricos; b) el $80 \%$ de los pacientes eran preescolares o escolares; c) los principales motivos de consulta fueron fiebre, síntomas digestivos y respiratorios; los cuales variaron según grupo etario, gravedad y estación del año; y, d) el riesgo de hospitalización fue más elevado en el grupo de $\mathrm{RN}$ y en aquellos que consultaron por ictericia, síntomas neurológicos o intoxicaciones.

El espectro de motivos de consulta descrito para cada rango etario y el bajo perfil de riesgo de los pacientes pediátricos en la admisión al SU son concordantes con los datos reportados en la literatura nacional e internacional $1^{5,14,17-21}$. Sin embargo, las consultas por lesiones traumáticas e intoxicaciones fueron menos frecuentes en nuestro estudio $(4,4 \%$ y $0,2 \%$, respectivamente) comparado con otros estudios internacionales $(20 \text { a } 40 \%)^{5,19,20,22}$, aunque similar a lo descrito en una cohorte de pacientes 
pediátricos atendidos en el Hospital Roberto del Río ${ }^{14}$. Esta diferencia podría estar determinada en parte por el hecho de que nuestra institución no es un centro de referencia de trauma y haber excluido del análisis a los pacientes atendidos en el SU de traumatología. Sin embargo, se requieren más estudios para aclarar la real incidencia de trauma e intoxicaciones en la población pediátrica de nuestro país.

La edad de los pacientes influyó en el motivo de consulta, la gravedad y el riesgo de hospitalización similar a lo descrito por Nelson y cols. ${ }^{23}$ y Goh y cols. ${ }^{19}$; siendo más frecuente la hospitalización de niños menores de un año. Los motivos de consulta variaron de acuerdo a la estación del año, predominando las consultas por fiebre y síntomas respiratorios en invierno y las consultas por fiebre y síntomas digestivos durante el verano.

La tasa de hospitalización fue sustancialmente menor en nuestro estudio comparado con la literatura nacional y extranjera $(4 \% v s$ $4,4 \%$ a $25 \%$ ) a pesar de tener un perfil de seve-

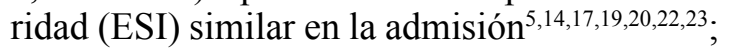
esta disparidad podría estar determinada por varios factores epidemiológicos, estructurales y administrativos. El HCPUC no es un centro de referencia de trauma y tiene una capacidad relativamente pequeña de camas pediátricas, lo que podría determinar la menor tasa de admisión. Los principales factores de riesgo de hospitalización fueron el período de $\mathrm{RN}$ y las consultas por ictericia, síntomas neurológicos e intoxicaciones. Finalmente, la tasa de consultas repetidas fue similar a lo comunicado en otros estudios $(3,9 \% \text { vs } 2,7-5,2 \%)^{24-28}$; lo cual puede ser explicado por el perfil sociodemográfico y clínico de los pacientes que consultan en nuestro SU, donde los médicos pediatras citan a control a los pacientes en el SU como alternativa a la hospitalización o a su derivación a los servicios de atención primaria.

Las principales limitaciones de nuestro estudio epidemiológico fueron la pérdida de registros clínicos (4\%), el desconocimiento de las comorbilidades y la incapacidad de realizar seguimiento a los pacientes ambulatorios u hospitalizados. Además, la ejecución en un solo centro asistencial limitaría la generalización de los resultados. A pesar de las limitacio- nes previamente descritas, creemos que esta información puede ser de utilidad en la planificación y asignación de recursos de salud y en la elaboración de los programas de formación de pregrado y postítulo de la especialidad.

\section{Referencias}

1.- Danzl DF, Munger BS: History of Academic Emergency Medicine. http://www.saem.org/publicat/chap1.htm

2.- Williams AL, Blomkalns AL, Gibler WB: Residency training in emergency medicine: the challenges of the 21st century. Keio J Med 2004; 53: 203-9.

3.- American Academy of Pediatrics Committee on Pediatric Emergency Medicine, American College of Emergency Physicians Pediatric Committee, Emergency Nurses Association Pediatric Committee. Joint Policy Statement-Guidelines for Care of Children in the Emergency Department. Ann Emerg Med 2009; 54: 543-52.

4.- Retezar R, Bessman E, Ding R, Zeger SL, McCarthy $M L$ : The effect of triage diagnostic standing orders on emergency department treatment time. Ann Emerg Med 2011; 57: 89-99.

5.- Niska R, Bhuiya F, Xu J: National Hospital Ambulatory Medical Care Survey: 2007 emergency department summary. Natl Health Stat Report 2010; (26): 1-31.

6.- Mintegi S, Shavit I, Benito J: REPEM group (Research in European Paediatric Emergency Medicine). Pediatric emergency care in Europe: a descriptive survey of 53 tertiary medical centers. Pediatr Emerg Care 2008; 24: 359-63.

7.- García G, Lafuente A, Jiménez L, González A, Páez P: The demand for emergency services at pediatric hospitals: a study of 4,858 cases treated at the emergency department of a pediatric hospital. An Esp Pediatr 1990; 32: 99-104.

8.- Millar KR, Gloor JE, Wellington N, Joubert GI: Early neonatal presentations to the pediatric emergency department. Pediatr Emerg Care 2000; 16: 145-50.

9.- McCollough $M$, Sharieff $G Q$ : Common complaints in the first 30 days of life. Emerg Med Clin North Am 2002; 20: 27-48.

10.- Solis DP, Vega RP, González NF, Fernández AI, Espuñes $S P$, Fanjul JL: Atención a neonatos en una unidad de urgencias pediátricas. Anales de Pediatría 2011; 59: 548.

11.- Calado CS, Pereira AG, Santos VN, Castro MJ, Maio $J F$ : What brings newborns to the emergency department?: a 1-year study. Pediatr Emerg Care 2009; 25: 
244-8.

12.- Ziv A, Boulet JR, Slap GB: Emergency department utilization by adolescents in the United States. Pediatrics 1998; 101: 987-94.

13.- Aros S: Consulta del recién nacido en el servicio de urgencia. Rev Chil Pediatr 2004; 75: 270-4.

14.- Méndez B, Herrera P, Guerra H, Dattas JP, Muñoz B, Velasco J: Estructura de la consulta pediátrica en el Servicio de Urgencia. Hospital Infantil Roberto del Río. Rev Chil Pediatr 2005; 76: 259-65.

15.- Miranda C, Martínez F, Fariña J, Mihovilovic C: Consultas en Servicio de Urgencia Hospital Roberto del Río: ¿Cuáles son las razones de esta preferencia por sobre el nivel primario de atención? Rev Ped Elec 2007; 4: 3-10.

16.- Gilboy N, Tanabe P, Travers D, Rosenau AM: Emergency Severity Index (ESI): A Triage Tool for Emergency Department Care, Version 4. Implementation Handbook, 2012 Edition. AHRQ Publication No. 12-0014. Rockvi1le, MD. Agency for Healthcare Research and Quality.

17.- Alpern ER, Stanley RM, Gorelick MH, et al: Epidemiology of a pediatric emergency medicine research network: the PECARN Core Data Project. Pediatr Emerg Care 2006; 22: 689-99.

18.- Krauss BS, Harakal T, Fleisher GR: The spectrum and frequency of illness presenting to a pediatric emergency department. Pediatr Emerg Care 1991; 7: 67-71.

19.- Goh AY, Chan TL, Abdel-Latiff ME: Paediatric utilization of a general emergency department in a developing country. Acta Paediatr 2003; 92: 965-9.
20.- Massin MM, Montesanti J, Gérard P, Lepage P: Spectrum and frequency of illness presenting to a pediatric emergency department. Acta Clin Belg 2006; 61: 161-5.

21.- Weir R, Rideout E, Crook J: Pediatric use of emergency departments. J Pediatr Health Care 1989; 3: 204-10.

22.- Pileggi $C$, Raffaele $G$, Angelillo IF: Paediatric utilization of an emergency department in Italy. Eur J Public Health 2006; 16: 565-9.

23.- Nelson D, Walsh K, Fleisher GR: Spectrum and frequency of pediatric illness presenting to a general community hospital emergency department. Pediatrics 1992; 90 : 5-10.

24.- Jacobstein CR, Alessandrini EA, Lavelle JM, Shaw $K N$ : Unscheduled revisits to a pediatric emergency department: risk factors for children with fever or infection-related complaints. Pediatr Emerg Care 2005; 21: 816-21.

25.- Goldman RD, Ong M, Macpherson A: Unscheduled return visits to the pediatric emergency department-oneyear experience. Pediatr Emerg Care 2006; 22: 545-9.

26.- Goldman RD, Kapoor A, Mehta S: Children admitted to the hospital after returning to the emergency department within 72 hours. Pediatr Emerg Care 2011; 27: 808-11.

27.- Alessandrini EA, Lavelle JM, Grenfell SM, Jacobstein $C R$, Shaw KN: Return visits to a pediatric emergency department. Pediatr Emerg Care 2004; 20: 166-71.

28.- Cho CS, Shapiro DJ, Cabana MD, Maselli JH, Hersh $A L$ : A national depiction of children with return visits to the emergency department within 72 hours, 2001-2007. Pediatr Emerg Care 2012; 28: 606-10. 